VOL. $72(2005) \quad$ [271-281]

\title{
EXISTENCE OF POSITIVE SOLUTIONS FOR NONLOCAL AND NONVARIATIONAL ELLIPTIC SYSTEMS
}

\section{Yujuan Chen and Honguun GaO}

In the paper we prove a result on the existence of positive solutions for a class of nonvariational elliptic system with nonlocal source by Galerkin methods and a fixed point theorem in finite dimensions. We establish another existence result by the super and subsolution method and a monotone iteration.

\section{INTRODUCTION AND MAIN RESULTS}

In this paper, we consider the positive solutions for a class of elliptic system with nonlocal source:

$$
\begin{cases}-\Delta u=f_{1}(x, u)\|v\|_{\alpha_{1}}^{p_{1}}, & x \in \Omega \\ -\Delta v=f_{2}(x, v)\|u\|_{\alpha_{2}}^{p_{2}}, & x \in \Omega \\ u>0, v>0, & x \in \Omega \\ u=0, v=0, & x \in \partial \Omega\end{cases}
$$

where $\Omega$ is a bounded domain in $R^{N}, N \geqslant 1$, with smooth boundary $\partial \Omega, p_{i}>0$, $1 \leqslant \alpha_{i} \leqslant \infty$ and $f_{i}, i=1,2$, satisfy some assumptions to be stated below.

This system can be used to describe the steady-state solutions of parabolic systems with a nonlocal source:

$$
\begin{cases}u_{t}-\Delta u=f_{1}(x, u)\|v\|_{\alpha_{1}}^{p_{1}}, & x \in \Omega, t>0 \\ v_{t}-\Delta v=f_{2}(x, v)\|u\|_{\alpha_{2}}^{p_{2}}, & x \in \Omega, t>0 \\ u(x, t)=0, v(x, t)=0, & x \in \partial \Omega, t>0 \\ u(x, 0)=u_{0}(x), v(x, 0)=v_{0}(x), & x \in \Omega .\end{cases}
$$

When $f_{1}, f_{2}$ are positive constants, system (1.2) has been discussed by Deng, Li and $\mathrm{Xie}$ (see [4]). It was proved that if $p_{1} p_{2}<1$ every nonnegative solution is global; whereas if $p_{1} p_{2}>1$, and the initial data is sufficiently large, the nonnegative solution of (1.2) blows up in finite time, and if the initial data is sufficiently small, the nonnegative solution of (1.2) is global.

Received 3rd May, 2005

This project was supported by the NSF of Jiangsu Education Office of PRC 03KJD1101690.

Copyright Clearance Centre, Inc. Serial-fee code: 0004-9727/05 \$A2.00+0.00. 
Over the last few decades, many physical phenomena were formulated into nonlocal mathematical models (see $[\mathbf{2}, \mathbf{3}, \mathbf{4}, \mathbf{5}, \mathbf{6}, \mathbf{7}]$ or references therein). The corresponding steady-state solutions have been attached great importance. Compared to the existence of positive solutions for elliptic problems, different techniques (variational methods, degree theory, fixed point theory, sub and supersolutions, Galerkin methods, moving hyperplane methods, et cetera) have been used (see for instance $[2,3,4,5,6,7]$ ). However, to the author's best knowledge, there is no literture on system (1.1). Since the system (1.1) has no variational structure, it is worthwhile to recall some of related nonvariational methods.

In [1], Alves and de Figureiredo used Galerkin methods and fixed point theorems in finite dimension and proved the existence of solutions of the local semilinear elliptic systems

$$
\begin{aligned}
& -\Delta u=a u^{\alpha}+f(x, u, v), \\
& -\Delta v=b v^{\beta}+g(x, u, v),
\end{aligned}
$$

in a bounded domain with homogeneous boundary conditions.

In [3] Correa studied the problem

$$
-a\left(\int_{\Omega}|u|^{q}\right) \Delta u=H(x) f(u)
$$

with homogeneous boundary condition. To prove the existence of positive solutions, the Krasnoselskii fixed point theorem has been used in the unidimensional case and the Schaefer fixed point theorem in the multidimensional case.

In [2] Correa, Silbano Menezes and Ferreira used fixed point theorems and index theory to establish a positive solution for the problem

$$
-a\left(\int_{\Omega}|u|\right) \Delta u=f(x, u)
$$

Furthermore, they proved another existence result by using sub and supersolutions without monotone iteration, and relying heavily on a comparison principle and the Schaefer fixed point theorem.

The purpose of this paper is to study the existence of solutions of (1.1) in two cases via

(a) Galerkin methods

(b) super and subsolution techniques,

respectively. Our work is motivated by $[1,2,3,6]$. From the viewpoint of physics, we are considering the positive weak solutions. We shall use the following definition of the weak solution for (1.1). 
DEFINITION 1.1: We say a function $(u, v) \in H_{0}^{1}(\Omega) \times H_{0}^{1}(\Omega)$ is a weak solution of (1.1) provided

$$
\begin{aligned}
& \int_{\Omega} \nabla u \nabla w d x=\|v\|_{\alpha_{1}}^{p_{1}} \int_{\Omega} f_{1}(x, u) w d x \\
& \int_{\Omega} \nabla v \nabla w d x=\|u\|_{\alpha_{2}}^{p_{2}} \int_{\Omega} f_{2}(x, v) w d x
\end{aligned}
$$

for all $w \in H_{0}^{1}(\Omega)$.

This paper is organised as follows. In the next section, we use Galerkin methods to establish an existence theorem for the solution for system (1.1) when $N \geqslant 3,0<p_{i}<1$, $1<\alpha_{i}<(2 N) /(N-1)$ or $N=1,2,0<p_{i}<1,1 \leqslant \alpha_{i}<\infty, i=1,2$. In Section 3, we established another existence result for (1.1) by making use of super and subsolution techniques and where the monotone iteration is used. When $f_{i}(i=1,2)$ is bounded and $p_{1} p_{2}<1$, we get a pair of super and subsolution of (1.1). Finally we consider the special case $\alpha_{i}=p_{i}=1, i=1,2$ and show the only probable solution is the trivial one under some assumptions on $f_{1}, f_{2}$.

Our main results read as follows.

THEOREM 1.2. If either the following (H1) or (H2) is satisfied, system (1.1) has a positive solution.

(H1) $N \geqslant 3,0<p_{i}<1,1<\alpha_{i}<(2 N) /(N-2)$,

$$
\begin{aligned}
0 & \leqslant D_{1}(x) \leqslant f_{1}(x, u) \leqslant A_{1}(x)|u|^{r_{1}}+B_{1}(x), \\
0 & \leqslant D_{2}(x) \leqslant f_{2}(x, v) \leqslant A_{2}(x)|v|^{r_{2}}+B_{2}(x), \\
B_{i}(x) & \in L^{\left(\alpha_{j}\right) /\left(\alpha_{j}-1\right)}, A_{i}(x) \in L^{\left(\alpha_{j}\right) /\left(\alpha_{j}-r_{i}-1\right)},
\end{aligned}
$$

$r_{i}<\min \left\{1-p_{i}, \alpha_{j}-1\right\}, D_{i}(x) \not \equiv 0(i=1,2)$, where $i j=2$ and $i, j$ are positive integers.

(H2) $\quad N=1,2$, for $i=1,2,0<p_{i}<1,1 \leqslant \alpha_{i}<\infty$.

THEOREM 1.3. Suppose that $f_{1}(\cdot, u) \in C(\bar{\Omega} \times R), f_{2}(\cdot, v) \in C(\bar{\Omega} \times R)$ are nondecreasing and Lipschitz continuous in $u$ and $v$ respectively. Assume there exists a weak supersolution $(\bar{u}(x), \bar{v}(x))$ and a weak subsolution $(\underline{u}(x), \underline{v}(x))$ of system (1.1), satisfying

$$
\begin{aligned}
& (\bar{u}(x), \bar{v}(x)) \geqslant 0,(\underline{u}(x), \underline{v}(x)) \leqslant 0 \text { on } \partial \Omega \text { in the trace sense, } \\
& (\underline{u}(x), \underline{v}(x)) \leqslant(\bar{u}(x), \bar{v}(x)) \text { almost everywhere in } \Omega .
\end{aligned}
$$

Then there exists a solution $(u, v)$ of (1.1), such that

$$
(\underline{u}(x), \underline{v}(x)) \leqslant(u(x), v(x)) \leqslant(\bar{u}(x), \bar{v}(x)) \text { almost everhwehre in } \Omega .
$$


Furthermore, if $(\underline{u}(x), \underline{v}(x))>0$ in $\Omega$, the solution of system (1.1) is positive.

THEOREM 1.4. In addition to the assumptions on $f_{1}, f_{2}$ of Theorem 1.3, we assume further that $0<m_{1} \leqslant f_{1}(x, u) \leqslant M_{1}, 0<m_{2} \leqslant f_{2}(x, v) \leqslant M_{2}$ in $\Omega \times R$, $p_{1} p_{2}<1$, then there exists a positive solution of system (1.1).

\section{GALERKIN METHODS}

In this section we show the existence of solutions for the system (1.1) via Galerkin methods. The following Fixed Point Theorem is used (see [1] or [6, Chapter 9]).

Proposition 2.1. Let $F: R^{K} \rightarrow R^{K}(K \in \mathbb{N})$ be a continuous function such that $\langle F(\xi), \xi\rangle \geqslant 0$ on $|\xi|=r$. Then, there exists $z_{0} \in \bar{B}_{r}(0)$ such that $F\left(z_{0}\right)=0$. Here $\langle\cdot, \cdot\rangle$ dentoes inner product of two vectors.

ProOF OF THEOREM 1.2: First, we shall study the existence for the solution of the following systems

$$
\begin{array}{ll}
-\Delta u=f_{1}(x, u)\|v\|_{\alpha_{1}}^{p_{1}}+\lambda \phi, & x \in \Omega, \\
-\Delta v=f_{2}(x, v)\|u\|_{\alpha_{2}}^{p_{2}}+\lambda \phi, & x \in \Omega, \\
u>0, v>0, & x \in \Omega \\
u=0, v=0, & x \in \partial \Omega .
\end{array}
$$

where $\phi \in C_{0}^{\infty}(\Omega)$ is a fixed positive function and $\lambda$ is a positive parameter.

Let $\sum=\left\{e_{1}, \ldots, e_{n}, \ldots\right\}$ be an orthogonal basis of the Hilbert space $H_{0}^{1}(\Omega)$ and be smooth. For each $m \in N$ define the subspace $V_{m}=\operatorname{span}\left\{e_{1}, \ldots, e_{m}\right\}$. It is well known that $\left(V_{m},\|\cdot\|\right)$ and $\left(R^{m},|\cdot|\right)$ are isometrically isomorphic by the natural linear map $T: V_{m} \rightarrow R^{m}$ given by

$$
v=\sum_{i=1}^{n} \xi_{i} e_{i} \mapsto T(v)=\xi=\left(\xi_{1}, \xi_{2}, \ldots, \xi_{m}\right) .
$$

So $\|v\|=|T(v)|=|\xi|$, where $|\cdot|$ and $\|\cdot\|$ denote the usual norms in $R^{N}$ and $V_{m}(\Omega)$, respectively.

Consider the following function $F: R^{2 m} \rightarrow R^{2 m}$ given by

$$
F(\xi, \eta)=\left(F_{1}(\xi, \eta), \ldots, F_{m}(\xi, \eta), G_{1}(\xi, \eta), \ldots, G_{m}(\xi, \eta)\right)
$$

where

$$
F_{i}(\xi, \eta)=\int_{\Omega} \nabla u \nabla e_{i} d x-\|v\|_{\alpha_{1}}^{p_{1}} \int_{\Omega} f_{1}(x, u) e_{i} d x-\lambda \int_{\Omega} \phi e_{i} d x
$$


and

$$
G_{i}(\xi, \eta)=\int_{\Omega} \nabla v \nabla e_{i} d x-\|u\|_{\alpha_{2}}^{p_{2}} \int_{\Omega} f_{2}(x, v) e_{i} d x-\lambda \int_{\Omega} \phi e_{i} d x, i=1,2, \ldots, m .
$$

In the above definitions we are using the identifications

$$
\xi \mapsto u=\sum_{i=1}^{m} \xi_{i} e_{i} \text { and } \eta \mapsto v=\sum_{i=1}^{m} \eta_{i} e_{i}
$$

Note that

$$
\begin{aligned}
\langle F(\xi, \eta),(\xi, \eta)\rangle=\|u\|^{2}- & \|v\|_{\alpha_{1}}^{p_{1}} \int_{\Omega} f_{1}(x, u) u d x \\
& -\lambda \int_{\Omega} \phi u d x+\|v\|^{2}-\|u\|_{\alpha_{2}}^{p_{2}} \int_{\Omega} f_{2}(x, v) v d x-\lambda \int_{\Omega} \phi v d x,
\end{aligned}
$$

and when $N \geqslant 3$, we have $H_{0}^{1}(\Omega) \subset L^{q}(\Omega)$, where $1<q<(2 N) /(N-2)$. By $(\mathrm{H} 1)$ and the Hölder inequality, we have

$$
\begin{aligned}
& \|v\|_{\alpha_{1}}^{p_{1}} \int_{\Omega} f_{1}(x, u) u d x+\|u\|_{\alpha_{2}}^{p_{2}} \int_{\Omega} f_{2}(x, v) v d x \\
& \leqslant\|v\|_{\alpha_{1}}^{p_{1}} \int_{\Omega}\left(A_{1}(x)|u|^{r_{1}}+B_{1}(x)\right) u d x+\|u\|_{\alpha_{2}}^{p_{2}} \int_{\Omega}\left(A_{2}(x)|v|^{r_{2}}+B_{2}(x)\right) v d x \\
& \leqslant\|v\|_{\alpha_{1}}^{p_{1}}\left[\left\|A_{1}(x)\right\|_{\alpha_{2} /\left(\alpha_{2}-r_{1}-1\right)}\|u\|_{\alpha_{2}}^{r_{1}+1}+\left\|B_{1}(x)\right\|_{\alpha_{2} /\left(\alpha_{2}-1\right)}\|u\|_{\alpha_{2}}\right] \\
& +\|u\|_{\alpha_{2}}^{p_{2}}\left[\left\|A_{2}(x)\right\|_{\alpha_{1} /\left(\alpha_{1}-r_{2}-1\right)}\|v\|_{\alpha_{1}}^{r_{2}+1}+\left\|B_{2}(x)\right\|_{\alpha_{1} /\left(\alpha_{1}-1\right)}\|v\|_{\alpha_{1}}\right] \\
& \leqslant C\left(\|u\|^{p_{1}+r_{1}+1}+\|v\|^{p_{1}+r_{1}+1}+\|u\|^{p_{2}+r_{2}+1}+\|v\|^{p_{2}+r_{2}+1}\right. \\
& \left.+\|u\|^{p_{1}+1}+\|v\|^{p_{1}+1}+\|u\|^{p_{2}+1}+\|v\|^{p_{2}+1}\right) .
\end{aligned}
$$

Here and elsewhere, we may use the same letter $C$ to indicate (possibly different) positive constants. With $\|(u, v)\|^{2}=\|u\|^{2}+\|v\|^{2}$, we obtain

$$
\begin{aligned}
\langle F(\xi, \eta),(\xi, \eta)\rangle \geqslant & \|(u, v)\|^{2}-C\left[\|(u, v)\|^{p_{1}+r_{1}+1}\right. \\
& \left.+\|(u, v)\|^{p_{2}+r_{2}+1}+\|(u, v)\|^{p_{1}+1}+\|(u, v)\|^{p_{2}+1}\right]-\lambda C\|(u, v)\| .
\end{aligned}
$$

As of $0<p_{i}+r_{i}<1$ and $0<p_{i}<1$, there exists a sufficient large $\rho$ such that

$$
\langle F(\xi, \eta),(\xi, \eta)\rangle>0 \text { on }\|(u, v)\|=\rho(\rho \text { is independent of } m) .
$$

It follows from Proposition 2.1 that, for each $m \in N$ there exists $\left(u_{m}, v_{m}\right) \in V_{m} \times V_{m}$ satisfying

$$
F\left(u_{m}, v_{m}\right)=(0,0), \quad\left\|\left(u_{m}, v_{m}\right)\right\| \leqslant \rho .
$$


Then (2.2) implies

$$
\begin{aligned}
& \int_{\Omega} \nabla u_{m} \nabla w d x=\left\|v_{m}\right\|_{\alpha_{1}}^{p_{1}} \int_{\Omega} f_{1}\left(x, u_{m}\right) w d x+\lambda \int_{\Omega} \phi w d x, \quad \forall w \in V_{m}, \\
& \int_{\Omega} \nabla v_{m} \nabla w d x=\left\|u_{m}\right\|_{\alpha_{2}}^{p_{2}} \int_{\Omega} f_{2}\left(x, v_{m}\right) w d x+\lambda \int_{\Omega} \phi w d x, \quad \forall w \in V_{m},
\end{aligned}
$$

with $\left\|u_{m}\right\|,\left\|v_{m}\right\| \leqslant \rho, \forall m \in N$. Let $u, v \in H_{0}^{1}(\Omega)$ be the weak limit of $\left\{u_{m}\right\}$ and $\left\{v_{m}\right\}$ respectively, choosing subsequences if necessary. So

$\left(u_{m}, v_{m}\right) \rightarrow(u, v)$ weakly in $H_{0}^{1}(\Omega)$,

$\left(u_{m}, v_{m}\right) \rightarrow(u, v)$ in $L^{q}(\Omega)$ for $1<q<\frac{2 N}{N-2}$, (since $H_{0}^{1}$ is compact in $L^{q}(\Omega)$ )

$\left(u_{m}, v_{m}\right) \rightarrow(u, v)$ almost everywhere in $\Omega$.

Considering $w \in V_{k}, \Psi \in V_{k}$ and $m \geqslant k$ we have

$$
\begin{aligned}
& \int_{\Omega} \nabla u_{m} \nabla w d x=\left\|v_{m}\right\|_{\alpha_{1}}^{p_{1}} \int_{\Omega} f_{1}\left(x, u_{m}\right) w d x+\lambda \int_{\Omega} \phi w d x, \quad \forall w \in V_{k}, \\
& \int_{\Omega} \nabla v_{m} \nabla \Psi d x=\left\|u_{m}\right\|_{\alpha_{2}}^{p_{2}} \int_{\Omega} f_{2}\left(x, v_{m}\right) \Psi d x+\lambda \int_{\Omega} \phi \Psi d x, \quad \forall \Psi \in V_{k},
\end{aligned}
$$

Then taking the limits as $m \rightarrow \infty$, we obtain

$$
\begin{aligned}
& \int_{\Omega} \nabla u \nabla w d x=\|v\|_{\alpha_{1}}^{p_{1}} \int_{\Omega} f_{1}(x, u) w d x+\lambda \int_{\Omega} \phi w d x, \quad \forall w \in V_{k}, \\
& \int_{\Omega} \nabla v \nabla \Psi d x=\|u\|_{\alpha_{2}}^{p_{2}} \int_{\Omega} f_{2}(x, v) \Psi d x+\lambda \int_{\Omega} \phi \Psi d x, \quad \forall \Psi \in V_{k},
\end{aligned}
$$

The equality (2.4) holds for all functions in $H_{0}^{1}$, as functions of the form $w$ and $\Psi$ are dense in this space. Hence $(u, v)$ is a weak solution of the system (2.1).

Recalling that $\lambda>0, \phi>0$, it follows from the maximum principle that $u, v>0$ in $\Omega$. Then according to (H1), we have

$$
\begin{aligned}
& -\Delta\left(u /\|v\|_{\alpha_{1}}^{p_{1}}\right) \geqslant D_{1}(x), \\
& -\Delta\left(v /\|u\|_{\alpha_{2}}^{p_{2}}\right) \geqslant D_{2}(x) .
\end{aligned}
$$

Let $\left(z_{1}, z_{2}\right)$ be the only positive solution of

$$
\begin{aligned}
-\Delta z_{1} & =D_{1}(x), \text { in } \Omega, \\
-\Delta z_{2} & =D_{2}(x), \text { in } \Omega, \\
z_{1} & =z_{2}=0, \text { on } \partial \Omega .
\end{aligned}
$$


According to the maximum principle, we have $u /\|v\|_{\alpha_{1}}^{p_{1}} \geqslant z_{1}, v /\|u\|_{\alpha_{2}}^{p_{2}} \geqslant z_{2}$ in $\Omega$, which implies

$$
\begin{aligned}
& \|u\|_{\alpha_{2}} \geqslant\left(\left\|z_{1}\right\|_{\alpha_{2}}\left\|z_{2}\right\|_{\alpha_{1}}^{p_{1}}\right)^{1 /\left(1-p_{1} p_{2}\right)}>0, \\
& \|v\|_{\alpha_{1}} \geqslant\left(\left\|z_{1}\right\|_{\alpha_{2}}^{p_{2}}\left\|z_{2}\right\|_{\alpha_{1}}\right)^{1 /\left(1-p_{1} p_{2}\right)}>0,
\end{aligned}
$$

So $\|u\|_{\alpha_{2}},\|v\|_{\alpha_{1}}$ has a positive lower bound. Moveover, take $\lambda=1 / n, n=1,2, \ldots$ and denote the corresponding solution of (2.1) as $\left(u_{n}, v_{n}\right)$, that is $\left(u_{n}, v_{n}\right)$ satisfies (2.4) with any $w$ and $\Psi$ in $H_{0}^{1}(\Omega)$. Set $w=u_{n}, \Psi=v_{n}$ in (2.4), we get

$$
\begin{aligned}
&\left\|\left(u_{n}, v_{n}\right)\right\|^{2} \leqslant C\left(\left\|\left(u_{n}, v_{n}\right)\right\|^{p_{1}+r_{1}+1}+\left\|\left(u_{n}, v_{n}\right)\right\|^{p_{2}+r_{2}+1}+\left\|\left(u_{n}, v_{n}\right)\right\|^{p_{1}+1}\right. \\
&\left.+\left\|\left(u_{n}, v_{n}\right)\right\|^{p_{2}+1}+\left\|\left(u_{n}, v_{n}\right)\right\| \cdot\|\phi\|_{2}\right) \text { for all } n \in N .
\end{aligned}
$$

Since $0<p_{i}+r_{i}<1$, and $0<p_{i}<1,\left\{\left(u_{n}, v_{n}\right)\right\}$ is a bounded sequence. As $n \rightarrow \infty$, thanks to the Sobolev embedding and the Lebesgue convergence theorem, a positive solution of (1.1) is obtained.

The Case (H2) can be proved similarly, since for $N=1$,

$$
H_{0}^{1}(\Omega) \subset C^{\alpha}(\bar{\Omega}), 0<\alpha \leqslant 1 / 2,
$$

and for $N=2$,

$$
H_{0}^{1}(\Omega) \subset L^{q}(\Omega), 1 \leq q<\infty
$$

So its proof is omitted.

\section{SUPER AND SUBSOLUTION METHOD}

Over the past two decades, supersolution and subsolution methods have been widely used in the proof of the existence of the solutions of parabolic or elliptic problems. In this section, we shall use this technique to prove the existence of the solution of system (1.1).

First, we shall give the definition of the super and subsolution of system (1.1)

Definition 3.1: A function $(\bar{u}, \bar{v}) \in H_{0}^{1}(\Omega) \times H_{0}^{1}(\Omega)$ is called a weak supersolution of (1.1) if

$\int_{\Omega} \nabla \bar{u} \nabla w d x \geqslant\|\bar{v}\|_{\alpha_{1}}^{p_{1}} \int_{\Omega} f_{1}(x, \bar{u}) w d x$, for each $w \in H_{0}^{1}, w>0$ almost everywhere, $\int_{\Omega} \nabla \bar{v} \nabla \Psi d x \geqslant\|\bar{u}\|_{\alpha_{2}}^{p_{2}} \int_{\Omega} f_{2}(x, \bar{v}) \Psi d x$, for each $\Psi \in H_{0}^{1}, \Psi>0$ almost everywhere. 
Similarly, $(\underline{u}(x), \underline{v}(x)) \in H_{0}^{1}(\Omega)$ is called a weak subsolution if it satisfies (3.1) with reversed inequalities.

Proof of Theorem 1.3: The method is standard, but for the reader's convenience, we complete it. Now write $\left(u_{0}, v_{0}\right)=(\underline{u}, \underline{v})$, we can construct a sequence $\left\{\left(u_{k}, v_{k}\right)\right\} \in H_{0}^{1}(\Omega) \times H_{0}^{1}(\Omega)$ from the following iterative process

$$
\begin{aligned}
& \int_{\Omega} \nabla u_{k} \nabla w d x=\left\|v_{k-1}\right\|_{\alpha_{1}}^{p_{1}} \int_{\Omega} f_{1}\left(x, u_{k-1}\right) w d x \\
& \int_{\Omega} \nabla v_{k} \nabla \Psi d x=\left\|u_{k-1}\right\|_{\alpha_{2}}^{p_{2}} \int_{\Omega} f_{2}\left(x, v_{k-1}\right) \Psi d x .
\end{aligned}
$$

We claim

$$
(\underline{u}, \underline{v}) \leqslant\left(u_{1}, v_{1}\right) \leqslant \cdots \leqslant\left(u_{n}, v_{n}\right) \leqslant \cdots \leqslant(\bar{u}, \bar{v}) .
$$

To confirm this, first note from (3.2) for $k=1$ that

$$
\begin{aligned}
& \int_{\Omega} \nabla u_{1} \nabla w d x=\left\|v_{0}\right\|_{\alpha_{1}}^{p_{1}} \int_{\Omega} f_{1}\left(x, u_{0}\right) w d x \\
& \int_{\Omega} \nabla v_{1} \nabla \Psi d x=\left\|u_{0}\right\|_{\alpha_{2}}^{p_{2}} \int_{\Omega} f_{2}\left(x, v_{0}\right) \Psi d x
\end{aligned}
$$

for each $w, \Psi \in H_{0}^{1}(\Omega)$. Subtract (3.4) from (3.1), recall $\left(u_{0}, v_{0}\right)=(\underline{u}, \underline{v})$ and set $w=\left(u_{0}-u_{1}\right)^{+}, \Psi=\left(v_{0}-v_{1}\right)^{+}$, to get

$$
\begin{gathered}
\int_{\Omega} \nabla\left(u_{0}-u_{1}\right) \cdot \nabla\left(u_{0}-u_{1}\right)^{+} d x \leqslant 0, \\
\int_{\Omega} \nabla\left(v_{0}-v_{1}\right) \cdot \nabla\left(v_{0}-v_{1}\right)^{+} d x \leqslant 0 .
\end{gathered}
$$

But

$$
\begin{aligned}
& \nabla\left(u_{0}-u_{1}\right)^{+}= \begin{cases}\nabla\left(u_{0}-u_{1}\right), & \text { almost everywhere on }\left\{u_{0} \geqslant u_{1}\right\}, \\
0 & \text { almost everywhere on }\left\{u_{0} \leqslant u_{1}\right\},\end{cases} \\
& \nabla\left(v_{0}-v_{1}\right)^{+}= \begin{cases}\nabla\left(v_{0}-v_{1}\right), & \text { almost everywhere on }\left\{v_{0} \geqslant v_{1}\right\}, \\
0 & \text { almost everywhere on }\left\{v_{0} \leqslant v_{1}\right\},\end{cases}
\end{aligned}
$$

(see [6, Chapter 5]). Consequently,

$$
\begin{aligned}
& \int_{\left\{u_{0} \geqslant u_{1}\right\}}\left|\nabla\left(u_{0}-u_{1}\right)\right|^{2} d x \leqslant 0 \\
& \int_{\left\{v_{0} \geqslant v_{1}\right\}}\left|\nabla\left(v_{0}-v_{1}\right)\right|^{2} d x \leqslant 0
\end{aligned}
$$


so that $\left(u_{0}, v_{0}\right) \leqslant\left(u_{1}, v_{1}\right)$ almost everywhere in $\Omega$. Now assume inductively

$$
\left(u_{k-1}, v_{k-1}\right) \leqslant\left(u_{k}, v_{k}\right) \text { almost everywhere in } \Omega
$$

From (3.2) we find similarly

$$
\begin{aligned}
\int_{\left\{u_{k} \geqslant u_{k+1}\right\}} \nabla\left(u_{k}-u_{k+1}\right) \cdot \nabla\left(u_{k}-u_{k+1}\right)^{+} d x & \\
& \leqslant \int_{\Omega}\left(f_{1}\left(x, u_{k-1}\right)\left\|v_{k-1}\right\|_{\alpha_{1}}^{p_{1}}-f_{1}\left(x, u_{k}\right)\left\|v_{k}\right\|_{\alpha_{1}}^{p_{1}}\right)\left(u_{k}-u_{k+1}\right)^{+} d x \leqslant 0, \\
\int_{\left\{v_{k} \geqslant v_{k+1}\right\}} \nabla & \left(v_{k}-v_{k+1}\right) \cdot \nabla\left(v_{k}-v_{k+1}\right)^{+} d x \\
& \leqslant \int_{\Omega}\left(f_{2}\left(x, v_{k-1}\right)\left\|u_{k-1}\right\|_{\alpha_{2}}^{p_{2}}-f_{2}\left(x, v_{k}\right)\left\|u_{k}\right\|_{\alpha_{2}}^{p_{2}}\right)\left(v_{k}-v_{k+1}\right)^{+} d x \leqslant 0 .
\end{aligned}
$$

The last inequality holds in view of (3.7) and $f_{1}(\cdot, u), f_{2}(\cdot, v)$ is nondecreasing in $u$ and $v$ respectively. Therefore $\left(u_{k}, v_{k}\right) \leqslant\left(u_{k+1}, v_{k+1}\right)$ almost everywhere in $\Omega$.

$$
\begin{gathered}
\int_{\left\{u_{k+1} \geqslant \bar{u}\right\}}\left|\nabla\left(u_{k+1}-\bar{u}\right)\right|^{2} d x \leqslant \int_{\left\{u_{k+1} \geqslant \bar{u}\right\}}\left(f_{1}\left(x, u_{k}\right)\left\|v_{k}\right\|_{\alpha_{1}}^{p_{1}}\right. \\
\left.-f_{1}(x, \bar{u})\|\bar{v}\|_{\alpha_{1}}^{p_{1}}\right)\left(u_{k+1}-\bar{u}\right)^{+} d x \leqslant 0, \\
\int_{\left\{v_{k+1} \geqslant \bar{v}\right\}}\left|\nabla\left(v_{k+1}-\bar{v}\right)\right|^{2} d x \leqslant \int_{\left\{v_{k+1} \geqslant \bar{v}\right\}}\left(f_{2}\left(x, v_{k}\right)\left\|u_{k}\right\|_{\alpha_{2}}^{p_{2}}\right. \\
\left.-f_{2}(x, \bar{v})\|\bar{u}\|_{\alpha_{2}}^{p_{2}}\right)\left(v_{k+1}-\bar{v}\right)^{+} d x \leqslant 0 .
\end{gathered}
$$

Next we show that $\left(u_{k}, v_{k}\right) \leqslant(\bar{u}, \bar{v})$ almost everywhere in $\Omega$. It is valid for $k=0$ by hypothesis (1.3). Assume now for induction $\left(u_{k}, v_{k}\right) \leqslant(\bar{u}, \bar{v})$ almost everywhere in $\Omega$. Then we have

Thus $\left(u_{k+1}, v_{k+1}\right) \leqslant(\bar{u}, \bar{v})$ almost everywhere in $\Omega$.

Therefore $(u(x), v(x)):=\lim _{n \rightarrow \infty}\left(u_{n}(x), v_{n}(x)\right)$ exists for almost all $x$. Furthermore, by the assumptions on $f_{i}(i=1,2)$, there is a subsequence $\left\{\left(u_{n_{j}}, v_{n_{j}}\right)\right\}_{j=1}^{\infty}$ which converges weakly in $H_{0}^{1}(\Omega) \times H_{0}^{1}(\Omega)$ to $(u, v)$. So, $(u, v)$ is a weak solution of (1.1). The theorem follows.

Theorem 1.4 is an example in which the above result applies.

Proof of ThEOREM 1.4: Let $\left(w_{1}, w_{2}\right)$ be the only solution of

$$
\begin{aligned}
& -\Delta w_{1}=m_{1}, \text { in } \Omega, \\
& -\Delta w_{2}=m_{2}, \text { in } \Omega, \\
& w_{1}=w_{2}=0, \text { on } \partial \Omega .
\end{aligned}
$$


Set

$$
\underline{u}=\left(\left\|w_{1}\right\|_{\alpha_{1}}^{p_{1} p_{2}}\left\|w_{2}\right\|_{\alpha_{2}}^{p_{2}}\right)^{1 /\left(1-p_{1} p_{2}\right)} w_{1}, \quad \underline{v}=\left(\left\|w_{2}\right\|_{\alpha_{1}}^{p_{1} p_{2}}\left\|w_{1}\right\|_{\alpha_{2}}^{p_{2}}\right)^{1 /\left(1-p_{1} p_{2}\right)} w_{2}
$$

then $(\underline{u}, \underline{v})$ is a positive subsolution of system (1.1). Similarly, if we denote by $\left(W_{1}, W_{2}\right)$ the only positive solution of (3.8) with $m_{1}, m_{2}$ be replaced by $M_{1}, M_{2}$ respectively, we can get the supersolution $(\bar{u}, \bar{v})$ of system $(1.1)$, where

$$
\bar{u}=\left(\left\|W_{1}\right\|_{\alpha_{1}}^{p_{1} p_{2}}\left\|W_{2}\right\|_{\alpha_{2}}^{p_{2}}\right)^{1 /\left(1-p_{1} p_{2}\right)} W_{1}, \quad \bar{v}=\left(\left\|W_{2}\right\|_{\alpha_{1}}^{p_{1} p_{2}}\left\|W_{1}\right\|_{\alpha_{2}}^{p_{2}}\right)^{1 /\left(1-p_{1} p_{2}\right)} W_{2}
$$

As $m_{i} \leqslant M_{i}$, we have $w_{i} \leqslant W_{i}, i=1,2$. In addition, $0<p_{1} p_{2}<1$, so $(\underline{u}, \underline{v}) \leqslant(\bar{u}, \bar{v})$. Theorem 1.3 completes the proof.

Now, for the the special case $\alpha_{i}=p_{i}=1$, becomes the system

$$
\begin{aligned}
& -\Delta u=f_{1}(x, u) \int_{\Omega}|v| d x, \quad x \in \Omega, \\
& -\Delta v=f_{2}(x, v) \int_{\Omega}|u| d x, \quad x \in \Omega, \\
& u=v=0, \quad x \in \partial \Omega .
\end{aligned}
$$

Denote by $\varphi_{0}(x)$ the unique positive solution of the linear elliptic problem

$$
-\Delta \varphi_{0}(x)=1, \quad x \in \Omega ; \quad \varphi_{0}(x)=0, \quad x \in \partial \Omega .
$$

Set $\rho=\int_{\Omega} \varphi_{0}(x) d x$. Thus, we have the following result.

Proposition 3.2. In addition to the assumptions on $f_{1}, f_{2}$ of Theorem 1.3, assume further $0<f_{i} \leqslant M_{i}(i=1,2)$ in $\Omega \times R$. Then nonegative solutions of (3.9) exist if $\rho^{2} \leqslant 1 /\left(M_{1} M_{2}\right)$.

Proof: Applying $\rho^{2} \leqslant 1 /\left(M_{1} M_{2}\right)$, we see that there exist large positive constants $K_{1}$ and $K_{2}$ such that

$$
M_{1} \rho \leqslant K_{1} / K_{2} \leqslant 1 /\left(M_{2} \rho\right) .
$$

Let $W(x)=K_{1} \varphi_{0}(x), S(x)=K_{2} \varphi_{0}(x)$, then $(W, S)$ is a pair of supersolution of (3.9). Furthermore, $(0,0)$ is its subsolution, so we can get a nonnegative solution via Theorem 1.3 .

Indeed, we have the following result.

Proposition 3.3. Assume $0<m_{i}<f_{i} \leqslant M_{i}(i=1,2)$ in $\Omega \times R$, and $\rho$ satisfies

$$
\rho^{2}<\frac{1}{M_{1} M_{2}} \text { or } \rho^{2}>\frac{1}{m_{1} m_{2}}
$$


Then the system (3.9) only possesses the trivial solution.

PROOF: Suppose $(u, v)$ is a non trivial solution of (3.9). Multiplying (3.9a), (3.9b) through by $\varphi_{0}$ and integrating on $\Omega$ respectively, we find

$$
\begin{aligned}
& \int_{\Omega} u d x=\|v\|_{1} \int_{\Omega} f_{1}(x, u) \varphi_{0} d x \\
& \int_{\Omega} v d x=\|u\|_{1} \int_{\Omega} f_{2}(x, v) \varphi_{0} d x .
\end{aligned}
$$

Since $0<m_{i} \leqslant f_{i} \leqslant M_{i}(i=1,2)$, and $\varphi_{0}$ is a positive function, we have

$$
\begin{aligned}
& m_{1} \rho\|v\|_{1} \leqslant\|u\|_{1} \leqslant M_{1} \rho\|v\|_{1}, \\
& m_{2} \rho\|u\|_{1} \leqslant\|v\|_{1} \leqslant M_{2} \rho\|u\|_{1} .
\end{aligned}
$$

So

$$
m_{1} m_{2} \rho^{2}\|u\|_{1}\|v\|_{1} \leqslant\|u\|_{1}\|v\|_{1} \leqslant M_{1} M_{2} \rho^{2}\|u\|_{1}\|v\|_{1} .
$$

The conclusion follows since (3.10) holds.

\section{REFERENCES}

[1] C.O. Alves and de D.G. Figureiredo, 'Nonvariational elliptic systems via Galerkin methods, function spaces, differential operators and nonlinear analysis', in The Hans Tridbel Anniversary Volume (Birkhauser Berlag Base 1, Switzerland, 2003), pp. 47-57.

[2] F.J.S.A. Correa, D.B. Silbano Menezes and J. Ferreira, 'On a class of problems involving a nonlocal operator', Appl. Math. Comput. 147 (2004), 475-489.

[3] F.J.S.A. Correa, 'On positive solutions of nonlocal and nonvariational elliptic problems', Nonlinear Anal. 59 (2004), 1147-1155.

[4] W.B. Deng, Y.X. Li and C.H. Xie, 'Blow-up and global existence for a nonlocal degenerate parabolic system', J. Math. Appl. 277 (2003), 199-217.

[5] P. Drabek and J. Hermandez, 'Existence and uniqueness of positive solutions for some quasilinear elliptic problems', Nonlinear Anal. 44 (2001), 189-204.

[6] L.C. Evans, Partial differential equations, Graduate Studies in Mathematics 19 (American Mathematical Society, Providence, R.I., 1998).

[7] R. Stanczy, 'Nonlocal elliptic equations', Nonlinear Anal. 47 (2001), 3579-3584.

Department of Mathematics

Nanjing Normal University

Nanjing 210097

Peoples Republic of China

\author{
Department of Mathematics \\ Nantong University \\ Nantong 226007 \\ Peoples Republic of China \\ e-mail: nttccyj@ntu.edu.cn
}

